Año 1 (2010) | artículo № 1 | Págs. 1 - 19

\title{
Consecuencias de una comunicación intrafamiliar marcada por estereotipos de género en el Consejo Popular Vivero
}

\author{
Consequences of a communication intrafamiliar marked by gender \\ stereotypes in the Popular Council Vivero
}

\author{
Lic. Gladys Caridad Seguí León \\ Universidad de Pinar del Río "Hermanos Saiz Montes de Oca" (Cuba) \\ gladysc@fcsh.upr.edu.cu \\ Recibido: 20 de Marzo de 2010 \\ Aceptado: 7 de junio de 2010
}

\begin{abstract}
Resumen
La realización de este trabajo demuestra cómo en un mismo país, incluso en una misma región pueden existir grandes diferencias en el desarrollo de la infancia en niños de una localidad determinada atendiendo a la comunicación intrafamiliar que se establece.

En una comunidad rural es totalmente distinta a una comunidad urbana aún con las mismas oportunidades. La atención médica y la educación en Cuba son absolutamente gratuitas, sin embargo hay elementos que se nos van quedando de la mano como la orientación familiar, el desarrollo local y la cultura de la región.

Es importante realizar un estudio periódico al desarrollo de la comunicación intrafamiliar y su repercusión en infancia en estas regiones campesinas pues la mayoría de los estudios sobre comunicación no giran precisamente alrededor de la repercusión en los niños, los pocos se realizan en la ciudad y se van quedando olvidadas localidades como ésta, dónde la violencia intrafamiliar y el machismo forman parte de la cotidianidad de la mayoría de estas familias.

La desorientación de la familia, en la crianza de los pequeños y el importante papel que desempeña la misma en el desarrollo de una infancia feliz, atenta contra el hombre y la mujer comprometidos que toda sociedad aspira.
\end{abstract}

\section{Summary}

They summarize: The realization of this work demonstrates how in oneself country, even in oneself region big differences can exist in the development of the childhood in children of a certain town assisting to the communication intrafamiliar that settles down.

In a rural community it is completely different to an urban community still with the same opportunities. The medical attention and the education in Cuba are absolutely gratuitous, however there are elements that are gone being of the hand like the family orientation, the local development and the culture of the region.

It is important to carry out a periodic study to the development of the communication intrafamiliar and their repercussion in childhood in these rural regions because most of the studies have more than enough communication they don't in fact rotate around the 
repercussion in the children, the few ones are carried out in the city and they go staying forgotten towns like this, where the violence intrafamiliar and the machismo are part of the cotidianidad of most of these families.

The disorientation of the family, in the upbringing of the small ones and the important paper that it carries out the same one in the development of a happy childhood, attentive against the man and the committed woman that all society aspires.

Palabras Clave: Rural, campesinos, escuela, educación, derechos, recreación, alimentación, morbilidad, parasitismo, asma.

Key Words: Rural, peasants, school, education, rights, recreation, feeding, morbilidad, parasitism, asthma.

\section{Introducción}

"No hay causa que merezca más alta prioridad que la protección y el desarrollo del niño, de quien dependen la supervivencia, la estabilidad y el progreso de todas las naciones y, de hecho, de la civilización humana".

Plan de Acción de la Cumbre Mundial a favor de la Infancia, 30 de septiembre de 1990.

La infancia es el período más sensible del desarrollo humano, los primeros años de vida son determinantes para el desarrollo físico, psíquico y social de la persona. El concepto de Infancia se diferencia del de menor y del de niñez, el concepto de menor priva al niño de su participación en las relaciones sociales, y aunque la participación debe ser adecuada para su edad, los niños y las niñas deben ser sujetos de derechos, donde con una orientación se busque que el infante tenga autonomía y conozca qué es mejor para sí mismo.

Los niños no comprenden sus Derechos y Deberes porque nosotros los adultos tampoco los comprendemos y no estamos generando contextos para transmitirlos y comunicarlos. Los adultos no sabemos asumir los Derechos y Deberes para que los niños y niñas tengan un adecuado modelo de convivencia familiar donde puedan ejercerlos. El niño es un sujeto con derecho a participar, a ser escuchado, tiene voz propia y sus intereses deben estar en función de sus necesidades, por encima de cualquier estimación o fenómeno social.

Una mirada puesta en la diversidad nos lleva a ver, en primer lugar, que eso que nosotros llamamos infancia no representa lo mismo ni es vivido de la misma manera en todos los grupos humanos. 
Pensando en la complejidad y diversidad de una sociedad nos obliga a preguntarnos ¿de qué hablamos cuando hablamos de niño o de infancia? El estudio antropológico de diferentes sociedades ha dado cuenta de la complejidad y variedad de las relaciones que pueden establecerse entre los procesos de crecimiento y desarrollo físico y la edad social. Siendo esos datos biológicos socialmente manipulables, los límites etéreos remiten a procesos de clasificación a través de los cuales en cada grupo social se instituyen las divisiones del mundo social, se hacen y se deshacen grupos, como la infancia, la adolescencia o la adultez (Bourdieu, 1983). Así, la niñez como grupo no comienza a existir como tal, para sus integrantes y para los demás, hasta que no es distinguida y recortada a través del conocimiento y reconocimiento de ciertas características que, al ser leídas como propias de una clase particular de personas, las transforma en una categoría social.

La familia es la primera escuela donde aprendemos cómo comunicarnos. La forma como aprendemos a comunicarnos en nuestra familia de origen determinará cómo nos comunicamos con los demás.

Así el niño comienza aprendiendo gestos y tonos de voz de sus padres y hermanos, comunicándose a través de ellos. Así las familias establecen formas de coordinarse que determinan y satisfacen las necesidades de todos sus miembros.

La forma de comunicarse que tienen los miembros de la familia, determinará la forma en que los niños que en ella crecen aprendan una manera de emocionarse y de pensar. Esto significa que cada familia enseña a través de la forma que tiene de comunicarse, su estilo particular; los valores, forma de pensar y mirar el mundo.

Por ello resulta de vital importancia el estudio de la comunicación intrafamiliar y sus consecuencias, pus la familia, sobre todo en el entorno rural marcada por estereotipos de género presenta factores que atentan contra el desarrollo de la infancia en Cuba.

La concepción de infancia, niñez, salud y comunicación intrafamiliar que tienen los habitantes del Consejo Popular Vivero dista mucho de la realidad. Por lo que el objetivo de este trabajo es:

Analizar los principales problemas que atentan contra el desarrollo de la infancia en los niños de la comunidad rural Vivero como consecuencia de una inadecuada comunicación intrafamiliar.

\section{Resultados}




\subsection{Importancia de la familia}

La familia tiene distintos niveles para comunicarse. De acuerdo a ello se establecen grupos que tienden a tener un cierto tipo de relación en su interior y con el otro grupo: nos referimos a los padres y los hijos. Al interior de cada uno de estos grupos existe una relación de igualdad. Esto es, los padres - ambos adultos - establecen una comunicación como padres entre ellos para ejercer mejor su función. A su vez, los hijos establecen una comunicación de hermanos, iguales, que les permite pasarlo bien, jugar y ejercer su rol de hijos frente a los padres.

La familia es el fundamento de toda sociedad bien construida, indispensable para el logro del bien común y además aparece como la unión más natural y necesaria a la comunidad; siendo además anterior a cualquier otra institución; es primera en el orden de la naturaleza, en relación con las demás agrupaciones en las que el hombre y la mujer se pueden encontrar.

Puesto que los padres han dado la vida a los hijos, están gravemente obligados a la educación de la prole y, por tanto, ellos son los primeros y obligados educadores. Este deber de la educación familiar es tan importante que, cuando falta, difícilmente puede suplirse. Es, pues, deber de los padres formar un ambiente familiar animado por el amor que favorezca la educación íntegra, personal y social de los hijos.

\subsection{Familia desde la pareja}

En un nivel distinto de comunicación se encuentra la pareja que constituye un espacio exclusivo de los adultos y que no tiene que ver con la crianza de los hijos, ni con la mantención de la casa. La pareja existe en torno a la comunicación de ellos como pololos, amigos y amantes.

De acuerdo a lo anterior, cuando hablamos de comunicación en la familia, debemos distinguir a qué nivel de estos sub-sistemas o grupos nos estamos refiriendo. Cuando la familia es capaz de dejar claros los límites y normas entre distintos grupos de la familia, la comunicación entre sus miembros tiende a ser más directa, eficaz y transparente. Esto significa que si los adultos en su rol de padres tienen un desacuerdo, deben ser capaces de no traspasar ese desacuerdo a la pareja y deben además tratar de no involucrar a los hijos en la discusión. Es algo que deberán resolver como padres, para luego transmitir a los hijos su decisión. En esto nada tiene que ver su comunicación como pareja.

El estilo y la forma de comunicación que tengan como familia o sub-grupo de esa familia, dependerá de su historia familiar y de su forma particular de relación.

Facultad de Ciencias de la Información - Universidad de La Laguna

Avenida César Manrique, s/n; Campus de Guajara

38071 La Laguna, Tenerife (Islas Canarias - España) 
A pesar de haberse visto constreñida con el desarrollo de la modernidad la familia sigue siendo insustituible como agencia primaria para la socialización del individuo y, en particular, para la estabilidad emocional de sus miembros. De todos los componentes de la estructura de cualquier sociedad ella es la más extendida y universal, está presente en todas las culturas y localidades. Que el niño o la niña vivan la niñez con calidad, dependerá en gran medida de la calidad del cuidado de la infancia, reconociendo a este como: Rol que se desarrolla a través de un conjunto de actividades específicas para la edad de la niñez durante el proceso de crianza que transcurre entre el nacimiento y el comienzo de la vida adulta, incluyendo el embarazo y el parto. En el ámbito familiar este cuidado se concreta en el marco de la relación paterno filial, en este cuidado tiene un papel primordial la familia, la cual está encargada de proporcionar al infante las condiciones socio materiales básicas para su desarrollo y el reconocimiento de sus necesidades de formación educativa. La familia es la encargada en el orden biológico de que la sociedad exista, y una de sus funciones más importantes y decisivas para el desarrollo de la humanidad es la educación y socialización de los individuos.

\subsection{Las reglas al interior de la Familia}

Las familias fijan reglas para convivir, que están de acuerdo a los valores y normas que los guían. Estas reglas por lo general son definidas y aplicadas por los padres. En algunas familias, y de acuerdo a su estilo y valores, los padres permiten que los hijos participen en la discusión sobre cierto tipo de reglas (permisos, salidas, paseos, deberes en la casa, etc..). El que los hijos participen o no de estas decisiones dependerá de la forma que cada familia tenga de ejercer el poder en su interior (más democrático o autoritario).

Lo fundamental es que quienes velen por el cumplimiento de estas reglas estén de acuerdo y hayan definido dichas normas en conjunto. Esto permitirá ser consistentes a los padres, aumentando su credibilidad y eficacia para lograr que las reglas se cumplan. Cuando los padres no logran acuerdo y tienden a discutir frente a los niños, o uno se alía con los hijos contra el otro, se tienden a generar problemas a nivel de la organización de la familia, que son difíciles de resolver (dictadura de los hijos, negación de la autoridad de uno de los padres, que dan origen a los habituales problemas conductuales en los hijos). Lo habitual es que los padres hagan uso de los castigos como forma de lograr que los hijos cumplan con las normas que se han fijado. En este sentido es necesario señalar que el uso del "costo de respuesta" (quitarles las cosas que les gustan frente a la trasgresión de las normas) brinda la mayor eficacia y eficiencia, como lo demuestran los estudiosos del tema, y que el premio y refuerzo (en forma contingente y estable) a las conductas deseadas es la forma más eficaz de lograr que se instauren nuevas formas de conducta en los hijos.

Facultad de Ciencias de la Información - Universidad de La Laguna

Avenida César Manrique, s/n; Campus de Guajara

38071 La Laguna, Tenerife (Islas Canarias - España) 


\subsection{Infancia y Niñez}

Una vez instituida como categoría, "la" infancia tiende a homogeneizar y enmascarar una gran variedad de experiencias de vida por las que atraviesan los niños/as concretos/ as, experiencias que justamente tienen que ver con la diversidad cultural y la desigualdad social. Categoría que, sin embargo, ya no aparece tan obvia ni unívoca: desde distintas perspectivas, diferentes autores han comenzado a plantear una crisis actual de esta noción de infancia propia de la modernidad occidental y a intentar dar cuenta de la pluralidad de caras de la niñez y adolescencia actuales La desnaturalización y particularización de la noción de infancia que de allí resulten nos llevará, entonces, a poner en tela de juicio ideas y acciones que se formulen en términos vagos de "lo mejor para el niño" (Rabello de Castro, 2001).

Atendiendo a lo planteado anteriormente sobre las diferencias entre infancia y niñez, la forma en que es vivida la infancia en los pequeños hoy en Cuba y el mundo, podemos decir que no muchos niños no disfrutan a plenitud su infancia.

Resulta común en la comunidad de Vivero, encontrar niños y niñas con un ritmo de vida más propio de la edad adulta que de la infancia. Los niños varones al salir de la escuela y los fines de semana andana caballo con los padres haciendo "cosas de hombres". Es muy común ver además que los niños y las niñas no comparten los mismos juegos: "eso es de macho", "las niñas pá la casa", "Las niñas juegan a las casitas y a las muñecas" "si sigues así vas a parecer un macho", "los hombres no lloran" "pareces una niñita", "deja la maric...(comportamiento vulgar de homosexuales)", "eres la pata del diablo", "el golpe enseña", "la letra con sangre entra" entre otras tantas son escuchadas a diario en esta comunidad, son frases comunes no hay nada de "particular" en ello, los gritos a los niños, los golpes y las palizas (aunque estas últimas con menos frecuencia) forman parte de la cotidianidad de la mayoría de estas familias que a pesar de todo cuidan (a su manera) de sus niños y se preocupan por ellos. El ambiente rural, la baja escolaridad de la mayoría de los habitantes ( 3 profesionales como promedio por zona) y la cultura patriarcal tan enraizada en los moradores de esta comunidad aún prejuiciosa entre otros factores, frenan el sano desarrollo de la infancia en los niños de Vivero.

No sólo se trata del desarrollo de la infancia, la educación de los padres es una problemática que traerá a la postre consecuencia nefastas ¿Qué pasará cuando esto niños crezcan? ¿Cómo será su comportamiento ante la vida? ¿Cómo trataran a sus mayores en un futuro? La educación de los hijos empieza por la educación de los padres. No se puede pedir hijos 
bien educados si ellos ven que los padres no lo están o si ven que no se comportan como deben. Los hijos ven muchos detalles en los padres que los padres creen que permanecen ocultos. Tienen una lupa especial para ver los defectos de los padres, procesan todo lo que ven, aunque después se callen. Enseguida descubren los puntos flacos de los padres y por donde conseguir hacer lo que quieren hacer. También ven los puntos fuertes, pero tratan de evitarlos para que no les produzcan contradicciones a sus intenciones y formas de comportamiento.

\subsection{Breve caracterización sociocultural de la comunidad Vivero}

El Consejo Popular Vivero se encuentra ubicado a escasos $2 \mathrm{Km}$. del pueblo San Juan y Martínez, que se encuentra a $21 \mathrm{Km}$. de la capital de la provincia Pinar del Río en Cuba.

Este Consejo popular cuenta con una escuela primaria, la escuela especial interna para niños con retraso escolar del municipio, un kiosco perteneciente a la cadena de Tiendas Recaudadoras de Divisas (TRD), un local para consultorio de la familia que agrupa dos consultorios con un mismo médico, un Despalillo de Tabaco y una Escogida de Tabaco, dos bodegas, una carnicería y una placita, no existe Círculo Social, ni Círculo Infantil, tampoco Sala de Video, ni Joven Club de Computación, no hay un parque infantil ni espacio para juegos y recreación como terreno de pelota o cancha para voleibol etc, no hay Teatro ni otros espacios que puedan ser utilizados por la comunidad, no existen Casas de Cultura, ni organismos de atención a la familia, salvo los esfuerzos realizados por el Médico de la Familia, la dirección de la escuela y la promotora del Programa Educa a tu Hijo. 
Figura № 1: Breve análisis demográfico del municipio San Juan y Martínez Año 2007

\begin{tabular}{|l|c|}
\hline Número de nacimientos & 636 \\
\hline Tasa de mortalidad infantil (por1000nacidos vivos) & $7,9 \%$ \\
\hline Tasa de mortalidad menores de 5 años & $7,9 \%$ \\
\hline Índice de bajo peso al nacer & $4.7 \%$ \\
\hline Población de 0 a 1 año & 677 \\
\hline Población de 1 años a 4 años & 2445 \\
\hline Población de 5 años a 14 años & 6872 \\
\hline Población de 14 años a 19 años & 5530 \\
\hline
\end{tabular}

Fuente: Elaboración Propia

Figura $\mathrm{N}^{\circ}$ 2: Caracterización socio demográfica de la población infantil en esta zona de acuerdo a los siguientes indicadores

\begin{tabular}{|l|c|c|}
\hline \multicolumn{2}{|c|}{$\begin{array}{c}\text { Análisis de algunos indicadores del Consejo Popular } \\
\text { Vivero (muestra) respecto al Municipio }\end{array}$} & $\begin{array}{c}\text { Respecto al total del } \\
\text { municipio }\end{array}$ \\
\hline Número de nacimientos & 13 & 2,04 \\
\hline Tasa de mortalidad infantil (por1000nacidos vivos) & 0 & - \\
\hline Tasa de mortalidad menores de 5 años & 0 & - \\
\hline Índice de bajo peso al nacer & 2 & 0,31 \\
\hline Población de 0 a 1 año & 6 & 0,88 \\
\hline Población de1años a 4 años & 21 & 0,85 \\
\hline Población de 5años a 14 años & 103 & 1,49 \\
\hline Población de 14 años a 19 años & 73 & 1,32 \\
\hline
\end{tabular}

Fuente: Elaboración Propia

\subsubsection{Población infantil por sexo y grupos de edades.}

El total de la población infantil analizada (muestra) es de 203 infantes, representando el $2,03 \%$ del total, dividido en cinco grupos de edades que conforman el período de la Infancia 
según la Convención de los Derechos del Niño. En la tabla 1 se muestra la división por edades y sexos.

\begin{tabular}{|c|c|c|c|}
\hline Grupo de edades & Femenino & Masculino & Total \\
\hline $0-1$ & 4 & 2 & 6 \\
\hline $1-4$ & 11 & 10 & 21 \\
\hline $5-9$ & 20 & 32 & 52 \\
\hline $10-14$ & 25 & 26 & 51 \\
\hline $15-19$ & 41 & 32 & 73 \\
\hline Total & 101 & 102 & 203 \\
\hline
\end{tabular}

Como se puede constatar el total de niños supera el de las niñas, representando un 50,24\% del total de la población infantil mientras que el de estas últimas representa un $49.75 \%$.

\subsubsection{Cobertura de enseñanza}

El Consejo popular Vivero perteneciente al municipio San Juan y Martínez, mantiene actualmente una cobertura de la atención primaria, secundaria y preuniversitaria de $100 \%$, casi todos los niños con edad entre los 5 y 19 años se encuentran vinculados al estudio, pues aunque representan la minoría (12 niños, 4 hembras y 8 varones) al terminan el preuniversitario (con 17 ó 18 años), no continúan estudios para dedicarse a negocios (que pueden ser legales o no, incluyendo el jineterismo), trabajar en el campo, casarse y convertirse en amas de casa (2 embarazadas) o vivir del ocio y lo que le den los padres (1 varón) para convertirse en un joven alcohólico.

\subsubsection{Tasa de mortalidad infantil}

La enfermera del consultorio, que atiende esta zona plantea que la población infantil este año no presentó casos de mortalidad en niños al nacer ni en menores de 5 años.

\subsection{4. Índice de bajo peso al nacer.}

La población infantil presenta algunos problemas con el bajo peso al nacer la "a pesar de los esfuerzos realizados por nosotros y explicarle a las futuras madres la importancia del cuidado de su alimentación y seguimiento por la a consultas", afirma la enfermera del 
consultorio tenemos este año de los 13 niños que nacieron 3 bajo peso al nacer 1 hembra y 2 varones, las madres de estos niños no sólo fueron mujeres que no se preocuparon lo suficiente durante su gestación sino que dos de ellas eran menores de 19 años, lo que evidentemente también representa un riesgo.

\subsubsection{Estado nutricional de los niños.}

La enfermera del consultorio que atienden esta zona plantean que la población infantil presenta algunos problemas con la nutrición, hay una niña desnutrida que ocupa el rango de 5 a 9 años, dos varones sobrepeso que ocupan el rango de 0 a 1 año y un varón obeso que ocupan el rango de 5 a 9 años. Los demás niños se encuentran en su peso normal.

2.5.6. Comportamiento de la morbilidad infantil por edades, sexo y tipos de enfermedades.

De manera general se puede observar que en el territorio analizado las patologías registradas (y las que no constan en registro pero son del dominio de la enfermera, quién además de vivir en la comunidad hace 16 años es enfermera del consultorio) son más frecuentes el parasitismo, el Asma Bronquial y las Enfermedades de la piel. En cuanto a las enfermedades respiratorias, específicamente el asma bronquial, en niños mayormente hijos de padres fumadores ( 2 niños varones en el rango de edades de 0 a 1 año) (en edades de un año en adelante 49 niños de ellos 30 varones y 19 niñas) "aquí todo el mundo fuma, en ocasiones los padres y miembros de la familia se despreocupan de la alimentación de los niños, por estar detrás del vicio del cigarro o tabaco y no es suficiente con las orientaciones y ayuda que se les brinda por parte nuestra" plantea la enfermera. El parasitismo, no existe registrado un dato específico en número pues son mayoría, pero un chequeo realizado por las autoridades de salud pública del Hospital de San Juan y Martínez a la escuela primaria Carlos Manuel de Céspedes de Vivero, constató que del número de niños en edades comprendidas entre 5 y 11 años se encuentran afectados el 93.2\% del total, la enfermera comenta que las enfermedades parasitarias tan comunes son provocadas por no hervir el agua, pero sobre todo por la tendencia que existe que los niños anden descalzos todo el tiempo. Las afectaciones de la piel son también muy comunes y aunque, de éstas últimas tampoco existe registro la enfermera plantea "son muchos casos los niños que tenemos con enfermedades de la piel". Tenemos una niña epiléptica, en el rango de edades de 1 a 4 años y un niño diabético en el grupo de 5 a 9 años. 
2.5.7. Cantidad y \% de niños que viven en viviendas en mal estado.

En el territorio existe una situación muy difícil con la vivienda, la comunidad, con casas de madera mayormente y de mampostería con techo de fibra fueron desbastadas por los huracanes Isidoro y Lili en el año 2002, a partir de entonces no han sido muchas las viviendas reparadas o totalmente construida y aún existen muchas familias viviendo en las llamadas "facilidades temporales". Al respecto la enfermera comenta "hija sólo mira a tu alrededor aquí todo el mundo vive en quimbos, y muchos con piso de tierra, no te digo vivienda en mal estado, te estoy hablando de casuchos en los que difícilmente puede vivir una familia, sobretodo numerosa como es la mayoría", existe un total de 47 niños viviendo en esas condiciones lo que representa $23,15 \%$ del total.

2.5.8. Cantidad y \% de niños que viven con padres u otro miembro de la familia alcohólicos.

Existen 8 niños que son hijos de padres alcohólicos, 3 que conviven con alcohólicos en la familia (1 abuelo, 2 tíos) para un total de 11 niños, que representa 5,41\% del total.

\subsubsection{Cantidad y \% de niños que viven en hogares con padres ausentes}

La ausencia de la figura paterna es un factor de incidencia en las familias de las comunidades la enfermera del consultorio plantea "ese es un problema grave pues aunque tenemos muchos niños, hijos de padres divorciados que desatienden a sus hijos y dejan toda la responsabilidad a la madre, tenemos algunas madres solteras que fueron abandonadas por el padre en las primeras etapas del embarazo y se desentendieron del bebé , otros padres, la mayoría, están de figura decorativa y lo que suceda con el niño tanto sus actividades escolares como su educación y salud son asunto de la madre, ellos no participan en nada " en esa situación ya sea por uno u otro caso tenemos en nuestra comunidad 57 niños, lo que representa $28,07 \%$ del total.

\subsubsection{Cantidad y \% de niños nacidos de madres menores de 19 años.}

Existen en la comunidad 18 niños hijos de madres menores de 19 años que representa 8,86 $\%$ del total. Estos niños son sanos y han tenido seguimiento especial por parte del médico de la familia, pediatra y ginecólogo y otros especialistas desde las primeras etapas del embarazo de las madres. 
2.5.11. Comportamiento de la lactancia materna por sexo. (Tiempo de lactancia exclusiva, acompañada con alimentos y motivos del abandono)

La lactancia exclusiva se plantea hasta los seis meses de vida del bebé, luego de este período se le pueden comenzar a introducir al niño alimentos ligeros, con la guía del médico de la familia, de los 18 lactantes que hay en la comunidad 16 madres han abandonado la lactancia exclusiva para un $88,88 \%$ de los lactantes, 14 niños (5 hembras y 9 varones) mantienen la lactancia acompañada con otros alimentos y 2 niños han sido privados de ella totalmente ( 1 niña y 1 niño) en el caso de la niña fue consultado y aprobado por el pediatra pues la madre epiléptica necesitaba comenzar a medicarse por las crisis y si lactaba no podía hacerlo, los demás casos comenzaron esta modalidad sin previa orientación de un especialista, los motivos que conllevan a esta decisión a juzgar por la enfermera del consultorio son los planteamientos de: el niño se queda con hambre, los varones son hartones y yo no tengo para más, no tengo leche en los senos, para que coja fuerza ,a mí me dieron puré a los 2 meses y mírame, entre otros. En el consultorio se les orienta sobre la importancia y ventajas de la lactancia y como estas conductas provocan un mal manejo de la alimentación del bebé, un proceso tan importante para el desarrollo de cualquiera de sus funciones vitales y actividades, más las madres hacen caso omiso.

\subsubsection{Cantidad y \% de niños inmunizados.}

En Cuba se realiza una labor profunda y sistemática en el tema de la vacunación, ningún niño se queda al margen de este programa. En el territorio que se analiza el $100 \%$ de los infantes está inmunizado, además el médico explica que es muy fácil la vacunación a través de la escuela, fundamentalmente la primaria a la cual van los médicos y enfermeras para cumplir con esta tarea. Es algo normal en la Isla que el sistema de salud se preocupe por la inmunización de su población siendo riguroso y oportuno. Además los padres se preocupan mucho de la vacunación de sus hijos.

\subsubsection{Cantidad y \% de niños que no acceden a agua potable sostenible.}

En el territorio analizado no existen problemas con el acceso al agua potable, ya que se abastece de un motor que bombea cada 2 días en tiempo muerto y diario en tiempo de zafra para facilitar el trabajo en el tabaco, esto beneficia también a las familias. Por su parte la enfermera del consultorio plantea que no se gana en conciencia de hervir el agua, para lograr un consumo más saludable, las personas lo ven como un trabajo extra e innecesario. 


\subsubsection{Cantidad y \% de niños accidentados.}

En esta comunidad ha habido accidentes por andar los niños en los caminos, así como con animales, ejemplo u niño mordido por un caballo, un pequeño de 2 meses que se le cayó a su hermanito de 3 años y recibió una herida en la cabeza de 3 puntos. Aunque se han dado más accidentes sólo estos 2 que representan el 0,98 \% del total están registrados en el consultorio, la mayoría de estos accidentes por descuido de los padres han sido desde heridas en los pies por corretear en las vegas de tabaco descalzos, caerse de las bicicletas, hasta ser pateados por bueyes, claro estos no aparecen y como tal no debe ser interés de la investigación, son datos que tengo como referencia por verlos a diario en la comunidad.

\subsection{Comunicación intrafamiliar en la localidad}

Todos los miembros de la familia poseen necesidades y expectativas de los otros respecto a la satisfacción de dichas necesidades. Una de las dificultades habituales de las familias radica en pensar que "el otro es capaz de adivinar lo que yo necesito y debe ser capaz de adivinar que hacer para ayudarme a satisfacer esta necesidad". Así mismo se espera que los otros adivinen los sentimientos y emociones.

Es fundamental que las familias sean capaces de aprender a lograr que sus miembros expresen sus necesidades, emociones y expectativas respecto a los otros, sin temor a la negación y descalificación por parte de los otros. Al explicitar dichos elementos se establecerán relaciones más claras y eficientes.

No es común en esta comunidad tener en cuenta el criterio de los niños porque "los niños no saben lo que quieren" no es cierta esta afirmación, la familia está en el deber de escuchar, proteger y respetar a los niños. Muchos miembros de esta comunidad caen en el mito de la capacidad de "adivinación" que pueden tener los otros miembros de la familia, y trabajar para que todos expresen sus emociones y necesidades a tiempo, evitando la acumulación de rabias y resolviendo los nudos de convivencia diaria, que afecta también a los pequeños.

En este proceso es conveniente trabajar para que cada uno se responsabilice por sus emociones y necesidades ("yo siento esta emoción", en vez de decir "ustedes me hacen sentir esto") evitando involucrar a los demás en las opciones que cada uno hace. Este tipo de aclaraciones permite crear un clima de confianza y aceptación que facilita la expresión de afectos y el logro de soluciones comunes. 


\subsubsection{Situación de la infancia en la localidad}

Con el objetivo de evaluar la situación de la infancia en la localidad se aplicó una entrevista a quien hasta hace 7 meses, fue el médico de la familia en la comunidad durante 14 años, este médico (actualmente director del Hospital de San Juan y Martínez) vivió en la comunidad, hasta hace 2 semanas, si agregamos que tiene dos niños que convivían con los en el resto de la comunidad y que el participaba con ellos en juegos y preparaba actividades en coordinación con la escuela lo convierte en la persona más conocedora de la situación que viven los niños en el Consejo Popular. La realización de esta entrevista tiene como objetivo: Evaluar la situación de la infancia en el Consejo Popular Vivero.

La entrevista consta de doce preguntas que fueron las más apropiadas para determinar el problema que más atenta contra el pleno desarrollo de los infantes de la Comunidad.

Se ofrecerán las respuestas dentro de la misma entrevista, para hacer más fácil su comprensión y análisis.

\section{Entrevista}

1. ¿Qué usted piensa de la situación de bienestar de los niños de su localidad?

Pienso que el bienestar de los niños de la comunidad es regular, pues la situación económica de casi la totalidad de las familias es muy modesta, el promedio de ingresos está alrededor de los $\$ 50.00$ mensual por persona de la familia y éstas son mayormente numerosas.

\section{2. ¿Considera usted que los niños del Consejo popular Vivero viven su infancia?}

La mayoría no vive su infancia, pues la localidad no tiene instalaciones deportivas ni recreativas, no hay parques de diversiones infantiles, no existen distracciones para que estos pequeños disfruten de una infancia plena, el juego es imprescindible en la infancia, desarrolla la imaginación y forma a los niños como hombres y mujeres de bien y útiles a la sociedad.

3. ¿A su juicio qué papel desempeña la familia de su localidad en el cuidado infantil?

Las familias cuidan de sus infantes, pero el nivel cultural de los padres y el ambiente social influyen y hasta determina en ocasiones que los niños no sean cuidados debidamente y por aquello de que "hijo varón a la calle" corran riesgo de accidente en los caminos y patios.

4. ¿Cómo es el estado de salud de los niños de Vivero? 
Las enfermedades más frecuentes en los niños de la localidad son el parasitismo, afectaciones respiratorias y enfermedades de la piel (en ese orden). Es común que los niños y niñas de la comunidad no usen zapatos y no tengan cultura de lavar las frutas antes de comerlas aunque se les eduque, en ese sentido la escuela los orienta mucho pero no es suficiente.

5. De los problemas de salud que presentan los niños de su comunidad ¿cuáles tienen relación con factores culturales?

A pesar d toda la información que reciben los padres y niños a través de diferentes medios los problemas que más nos golpean e inciden desde la cultura de la localidad son el parasitismo y los accidentes, a veces encontramos niños, casi siempre descalzos pues algunos usan zapatos sólo para ir a la escuela y no es por carecer de el calzado, son las 6:00 p.m. y están mudando caballos solos porque el padre se los enseña desde antes de aprender a caminar, muchos de los accidentes que tenemos hoy es causado por estas irresponsabilidades de los padres y por su puesto con niños varones, he visto niños con 5 días de nacidos ser traídos al hospital en un carro de caballo, eso es muy común aquí.

6. ¿Usted cree que las familias establecen diferencias entre las niñas y los niños que pueden provocar situaciones de salud?

No, las familias siempre cuidan con mucho celo del más enfermo, no con diferencias de género, al enfermo es al que mejor alimentan y atienden sin descuidar de los demás, dentro de lo mucho o poco que haya en cada hogar los niños en general son tratados de la misma manera, reciben el mismo cuidado y la misma alimentación.

7. ¿Por lo general los padres golpean más a los niños que a las niñas?

Los varones son más intranquilos, son más fuertes e impositivos, desde pequeños suelen usar esas libertades que les dan los padres y aunque no en todos los casos es posible que por lo mismo sean golpeados y castigados con mucha mayor frecuencia que las niñas los varones suelen ser más agresivos y rebeldes, precisamente por estas diferencias en la crianza puede que se sientan superiores a las niñas.

8. ¿Las niñas se quedan en la casa mientras los varones andan en el camino?

Las niñas por lo general permanecen en el hogar junto a la madre, generalmente los padres (padre y madre) cuidan de proteger a las niñas de los riesgos que corren fuera del hogar.

9. ¿No corren también los niños este riesgo? 
Sí, pero para los padres es diferentes ellos hacen cosas de "hombres", verás el riesgo forma parte de ser criados como hombres, la aventura, pasar trabajo, es de "hombres", aunque permíteme aclararte que no se hace consciente, es decir ningún padre expone a su hijo al peligro porque cree que será bueno, simplemente cree que andar conciertas libertades y haciendo tareas de adulto lo formarán como un hombre más completo, a las niñas las mantienen en la casa seguras junto a la madre ayudando a barrer, limpiar, para ser mañana "una buena mujer para una casa".

10. ¿Los niños varones quedan entonces descuidados en los caminos hasta altas horas de la noche?

No es en todos los casos, sólo en algunas excepciones, como dije anteriormente, los padres de Vivero cuidan de sus hijos de la forma que ellos consideran la más apropiada, los padres son celosos en el cuidado de sus niños aunque la forma no sea la mejor, al anochecer ya la mayoría de los niños están en sus casas.

11. ¿Ha existido algún accidente producto del descuido de los niños varones en los caminos?

Sí, aunque no el número exacto, conozco de varios accidentes sucedidos en la comunidad, sobre todo con niños varones, por andar en los caminos.

12. ¿Considera que las familias de Vivero crean estrategias (ya sean legales o no) para dar solución a los problemas de la vida cotidiana, como es la alimentación de los niños, o se mantienen en una posición más inactiva?

Creo en este sentido que existen cuatro tipos de familias en la comunidad. Las familias que logran darle solución a estos problemas por diversas vías y se preocupan por el sano desarrollo del infante, estas son una minoría bastante escasa. Las familias que hacen lo posible por cuidar y alimentar a sus hijos, pero a pesar de estos esfuerzos estoy convencido de que no son suficientes para el buen desarrollo y crecimiento de los niños. Existen familias que mantienen una posición más pasiva o inactiva ante estos problemas de la cotidianidad. Familias en las que los padres malgastan el dinero en fiestas, alcohol, cigarro u otros vicios olvidando así lo que es indispensable para la casa y con ello para sus hijos.

\subsubsection{Reflexiones sobre la comunidad}

Hace tiempo se analizan aspectos de la comunidad que nadie parece notar últimamente, elementos siempre fueron vistos como normal por los miembros del Consejo Popular Vivero. Por ejemplo la inmensa mayoría de los niños y niñas andan descalzos, es común en esta comunidad encontrar niños de 1 año en adelante hasta los 19, descalzos todo el día y parte de la noche andando por en los charcos y jugando con la tierra. 
Es imprescindible educar a los hijos para que la sociedad no les castigue cuando sean hombres. Si los padres no han dado a sus hijos la educación adecuada, no se han comunicado debidamente con ellos, o estos no la han asimilado, cada vez será más difícil convencerles de que tienen que cambiar y dejar a un lado esa rebeldía que empiezan a practicar, todo es cuestión que los padres estén bien formados, dediquen mucho tiempo y sobre todo mucho cariño hacia sus hijos, principalmente a los que han empezado con problemas. En manos de los padres está salvarlos o condenarlos cara al futuro.

Sin embargo más allá de la crianza que reciben estos niños, más allá de la cultura que atenta contra el adecuado desarrollo de la infancia en la localidad convirtiéndolos en espejos de sus progenitores esclavos de una cultura patriarcal, machista y extremadamente perjudicial para el desarrollo de la niñez plena, marcándola con tabúes y prejuicios.

Se encuentran niños que no tienen un espacio para jugar pelota, no tienen un espacio para la recreación excepto el pequeño patio de la escuela, creo que debemos mirar también un poco más a nuestro alrededor, amén de la crianza que reciban si es adecuada o no ¿cómo no van a pelear entre ellos si no tienen como combatir el ocio? No tienen biblioteca, sala de video ni otro centro que tribute a florecer su imaginación, no se realizan ferias del libro ni nada semejante. Siendo así no sólo hace falta una buena orientación a la familia que cada vez se vuelve más difícil de manejar y ninguna institución se ha ocupado de orientar, las autoridades como el gobierno y el Partido deben dirigir la mirada hacia esta comunidad y otras tantas que carecen de lo esencial para que un niño crezca sano y feliz pues el juego y la lectura es tan importante para el niño como el alimento y el sueño.

\section{Conclusiones}

Mucho se ha dicho que la base de la familia es el amor, pero a ese amor hay que alimentarlo y mantenerlo con una buena comunicación. Se debe trabajar también por el logro de la erradicación de la cultura patriarcal en nuestra sociedad y aún más en esas comunidades rurales dónde mujeres y niños son los mayores afectados y están desorientados presos aún del patriarcado y una comunicación intrafamiliar casi nula, es necesario el trabajo conjunto de Casa de orientación a la mujer (que no existe en esta comunidad) y de la Federación de Mujeres Cubanas para salvar estas familias, que se continúe trabajando sobre esta temática, considerando el sistema familiar como un laboratorio natural de aprendizaje, experimentación y transformación del ser humano.

En el caso específico de la comunicación entre padres e hijos, los problemas aumentan entre más libertad desean los hijos y más control quieren ejercer los padres. Los hijos quieren conocer y disfrutar el mundo, pero los padres los quieren proteger de él El tema cuidado 
infantil emerge en esta comunidad con la necesidad de erradicar el factor "sexismo" tan enraizado en los habitantes de la misma y que puede constituir una violación a los derechos del niño. Los padres de familia no reciben instrucciones adecuadas de cómo dar el debido cuidado a sus hijos, y cómo comunicarse debidamente con ellos. No conocen ni respetan el derecho del niño a vivir una infancia saludable.

Los padres deben comprender que los hijos son niños aún, pero ellos tienen opinión, y se les debe de respetar lo que piensen, porque ellos serán los únicos que construyan el camino de su vida y los padres deben entonces darles las herramientas y el apoyo.

Los niños no viven su infancia por varios factores, sobre todo culturales que les provoca enfermedades como el caso del parasitismo, por andar descalzos todo el tiempo, el asma bronquial por convivir con fumadores y los accidentes por estar en los caminos realizando labores de adultos o vagabundeando, el caso es "el macho al camino".

Otro factor que atenta contra el desarrollo de la infancia en los niños es la poca preocupación de algunos padres por su alimentación.

No todo es culpa de la cultura de la comunidad, es necesario también para estos niños contar con un lugar para su recreación y empleo de tiempo libre ya sea una sala de video, parque o instalación deportiva.

\section{Bibliografía}

1. Anuario Estadístico, 2007. En: Municipio San Juan Y Martínez

2. Barcia, M. 2003. La Familia Historia de su Historia. En: Vera, A. (comp.) La familia y las Ciencias Sociales. La Habana. Centro de Investigación y desarrollo de la Cultura Cubana Juan Marinello.

3. Brannen, J. Brien, M. 1996. Children in Families: Research and Policy, London, Falmer Press; James, Allison y Prout, Alan (ed.) (1997) Constructing and Reconstructing Childhood: Contemporary Issues in the Sociological Study of Childhood, London, Falmer Press. En: Bibliografía digital del Diplomado Violencia Intrafamiliar y estrategias de solución a las dificultades de la vida cotidiana en la familia cubana. UH, cátedra de la UNESCO. La Habana

4. Convención sobre los Derechos del Niño.1989. UNICEF

5. Entrevistas a expertos.

6. Fleitas, R. 2004. La familia cubana hoy. Las familias monoparentales. En: Mansson, S; Proveyer, C. Trabajo Social en Cuba y Suecia. Ediciones Arcadia.

7. Fleitas, R 2005. La Sociología de la Familia en Cuba. En: Selección de Lecturas de Sociología y Política Social de la Familia. Editorial Félix Varela. La Habana. p 287. 
8. Fleitas, R. 2008. Conferencia Familia y cuidado de la infancia. El modelo de salud. En: Diplomado Violencia intrafamiliar y estrategias de solución a las dificultades de la vida cotidiana en la familia cubana. UH, cátedra de la UNESCO. La Habana.

9. Fleitas, R. 2008. Conferencia Bienestar y calidad del cuidado de la infancia. El modelo de salud. En: Diplomado Violencia intrafamiliar y estrategias de solución a las dificultades de la vida cotidiana en la familia cubana. UH, cátedra de la UNESCO. La Habana.

10. Informe Mundial de la Infancia, 2007.

11.http://cheloveiga.espacioblog.com

12.http://www.innovacantabria.com

13.http://www.unicef.org

14.http://foro.univision.com

15.http://blog.micumbre.com

16. http://www.paginasprodigy.com/peimber/BOURDIEU.pdf

\section{Forma de citar este artículo en bibliografías}

Gladys Caridad Seguí León (2010): "Consecuencias de una comunicación intrafamiliar marcada por estereotipos de género en el Consejo Popular Vivero", en Revista PANGEA, 1, páginas 1 a 19. Red Académica Iberoamericana de Comunicación. Recuperado el _ de de 2 de: http://revistapangea.org/2010/09/13/01-01-101/ 